\title{
SCIENTIFIC Discovery: THAT-WhatS AND What-ThatS
}

\author{
SAMUEL SCHINDLER
}

Centre for Science Studies, Department of Mathematics, Aarhus University, Denmark

\begin{abstract}
In this paper I defend Kuhn's view of scientific discovery, which involves two central tenets, namely (i) that a scientific discovery always requires a discovery-that (i.e., the observation of $\mathrm{X}$ ) and a discovery-what (i.e., the correct conceptualisation of $\mathrm{X}$ ); and (ii) that there are two kinds of scientific discovery, resulting from the temporal order of the discovery-that and the discovery-what. I identify two problems with Kuhn's account and offer solutions to them from a realist stance. I also discuss alternatives to Kuhn's account.
\end{abstract}

\section{Introduction}

What is a scientific discovery? One of the first modern philosophers of science to systematically investigate this question was Kuhn (1959, I962b, I962a). One of the central points made by Kuhn is that a discovery always involves not only a discovery-that (the observation of the discovered object) but also a discovery-what (the correct conceptualisation of the discovered object); one without the other is insufficient for a discovery. What is less known is that Kuhn also distinguished between two broad classes of discovery: discoveries in which the discovery-that is made before the discovery-what (one may refer to those discoveries as that-what discoveries), and vice versa, discoveries in which the discovery-what is made before the discovery-that (what-that discoveries). Each of these classes of discoveries comes with distinctive features. It is the main purpose of this paper to bring to the attention of philosophers the strength of Kuhn's distinction between that-what and what-that discoveries.

The philosophical literature on scientific discovery, understood as the discovery of natural kinds, is not very sizable. Apart from the pioneering work by Kuhn-

Contact: Samuel Schindler <sks@css.au.dk> 
inspired by Hanson (1958) - there has only been a handful of more recent contributions (Achinstein 200I; Hudson 200I; McArthur 20II). ${ }^{\mathrm{I}}$ Those more recent accounts have all downplayed either the discovery-that or the discovery-what. This paper will show that these alternative accounts are too weak: they make scientists who are not discoverers into discoverers. On the other hand, this paper will make more precise an aspect of Kuhn's account, which has been left vague. By doing so, this paper will depart significantly from Kuhn's overall account of paradigmrelativity and show that some essential elements of Kuhn's account of discovery can be put to good use in a realist framework.

The structure of this paper is as follows. Section 2 will introduce Kuhn's account in detail. Section 3 will point to two issues in Kuhn's account: a conceptual and a descriptive one. Section 4 will address the descriptive problem by presenting two examples of important scientific discoveries that support Kuhn's account. Section 5 will propose a fix to the conceptual problem. Section 6 will briefly explore the extension of Kuhn's account to other entities than natural kinds. Section 7 will discuss three alternative accounts of scientific discovery. Section 8 will conclude the paper.

\section{Kuhn on Scientific Discovery}

For Kuhn, "discovering a new sort of phenomenon is necessarily a complex event, one which involves recognizing both that something is and what it is" (Kuhn I996: 55 ). It would be a mistake, according to Kuhn, to "assimilate" discoveries in science to the (naively construed) act of seeing or to other sense perceptions (Kuhn I996: 55). Rather a discovery, for Kuhn, not only involves the observation of an object, but also the correct conceptualisation of that object. Kuhn's main example for illustrating this point is the discovery of oxygen. Although Joseph Priestley was arguably the first to have isolated oxygen, ${ }^{2}$ he did not conceptualise it correctly. Rather, working within the theoretical framework of the phlogiston theory, Priestley thought that he had discovered dephlogisticated air, i.e., air depleted of phlogiston. Lavoisier, according to Kuhn, can not be said to have discovered oxygen either, because his conception of oxygen was also mistaken: he believed that oxygen gas was a combination of oxygen (i.e., the 'principle' of acidity) and ca-

I. In the philosophy of science the term 'discovery' is sometimes associated with the notion of 'context of discovery', which, somewhat paradoxically, concerns not so much the discovery of the phenomena, but rather the invention of theories. More specifically, in the context of discovery one asks about the reasons why certain scientists come up with certain theories. This paper has got nothing to say about this issue.

2. Another candidate is the Swedish apothecary C. W. Scheele, whose work on oxygen was not published until after the discovery of oxygen had been announced (Kuhn I996: 53). 
loric, the (non-existent) matter of heat. Without the requirement of the correct conceptualisation of the thing discovered, we would have to say that oxygen was discovered by anybody who first bottled impure oxygen, for Priestley himself did not manage to isolate a pure sample of oxygen (54). All we can say then, according to Kuhn, is that oxygen was discovered sometime in the period of I774 until I 777 . More generally, discoveries are "not isolated events, but extended episodes" where it is largely arbitrary to identify any one scientist as the discoverer of a scientific object (Kuhn I996: 52).

Interestingly, in a paper published in Science in 1962 (reprinted in Kuhn 1977), which formed the basis for chapter six of The Structure of Scientific Revolutions (Kuhn I996), Kuhn drew a distinction between two basic kinds of discoveries, which (for some reason) he no longer makes explicitly in The Structure. In one kind of discovery, the conceptualisation is carried out before the object in question is observed: these are classic cases of prediction, ${ }^{3}$ such as the discovery of missing elements in the periodic table, the neutrino, and radio waves (Kuhn I977: I66-7). But given that that these discoveries were anticipated (usually, but not always, on theoretical grounds), they are "an occasion only for congratulations, not for surprise". They are thus prime examples of normal science activity, which does not aim for surprising novelties (Kuhn I 996: 58 ). ${ }^{4}$ In contrast, in discoveries of the second kind, the conceptualisation of the thing discovered follows the observation of the thing discovered. Those discoveries may be referred to as that-what discoveries. It is those discoveries that Kuhn considered "troublesome" and which he made the main focus of chapter six in The Structure.

According to Kuhn, that-what discoveries have sharply distinct characteristics from what-that discoveries. Whereas what-that discoveries are instantaneous with regard to the incidence of the discovery-that (Kuhn I977: I7I), 5 only rarely give rise to priority debates (Kuhn I977: I66-7), and where, accordingly, "only a paucity of data can prevent the historian from ascribing [discoveries] to a particular time and place" (Kuhn 1977: 167), the contrary is the case in that-what discover-

3. Prediction need not be understood as strict deduction from a mathematized theory. It can be much weaker than that. See e.g., Scerri and Worrall (200I).

4. Sometimes Kuhn speaks as if what-that discoveries cannot be discoveries of new kinds of phenomena. His reason for this view is that in those discoveries "our conceptual categories are prepared in advance" to the phenomena in question (I996: 55-6, cf. 6I). However that is a mistake. Medeleev prepared the chemical community conceptually for new chemical elements when predicting them. When these chemical elements were discovered, they were clearly new kinds of elements (with respect to the knowledge of that time). What Kuhn rather seems to mean when he speaks in the above way is that what-that discoveries can never be unexpected or surprising discoveries (with regard to the knowledge of the time).

5. Verbatim, Kuhn says that only in what-that discoveries "can discovering that and discovering what occur together in an instant" (I977: I7I). Kuhn is most plausibly interpreted as judging the question of whether or not a discovery possesses a time dimension relative to the incidence of the discovery-that. 
ies. In that-what discoveries (such as in the discovery of oxygen), there necessarily is a time-dimension to discoveries, for it simply must take time to conceptualise correctly a thing for which one had no, or only an inapppropriate, conception at the time of observation (Kuhn I996: 55). Again, in contrast, in what-that discoveries the discovery simpliciter usually coincides with the discovery-that, because the correct conceptualisation has already been carried out before the new object is observed. The necessary time dimension of that-what discoveries, ${ }^{6}$ regularly involving several individuals, is therefore a major reason for why an attribution of a discovery to any one individual is "often impossible" and to a moment in time is "always imposssible" (Kuhn I996: 55). In that-what discoveries there are thus "no benchmarks to inform either the scientist or the historian when the job of a discovery has been done" (I977: I67). Although not all discoveries may fall neatly in either the what-that or the that-what category (I977: I67, n. 3 on I67), Kuhn clearly thinks that most cases do. It is to be noted, however, that Kuhn at least implicitly (by the choice of examples) restricts his discussion of discoveries to discoveries in the natural sciences (excluding mathematics). Moreover, he seems to be mainly interested in the discovery of natural kinds (rather than, e.g., laws). We shall follow Kuhn in these restrictions throughout this paper.

What Kuhn is also quite clear about is that his concern is "not at all" a practically useful account of discovery, which would help to disentangle priority debates. Instead he is interested first and foremost in the "nature of discovery". Nevertheless, probing answers to questions about priority, Kuhn points out, may help us to shed light on the nature of discovery, primarily by standard answers of the form "scientist $\mathrm{S}$ discovered object $\mathrm{O}$ at time t" turning out to be untenable (Kuhn I996: $54)$.

Lastly, for Kuhn, a discovery-that never proceeds conceptually unmediated. When we approach the phenomenological world, we always do so against the backdrop of some theories, ideas, or background assumptions. Also that-what discoveries do not happen in empty conceptual space. What critically distinguishes them from what-that discoveries, however, is that they are unexpected by the lights of the available conceptual resources. Now, if those conceptual resources happen to be part of a paradigm—as for Kuhn they usually are in normal science-then a violation of these assumptions through a newly observed phenomenon may lead to a paradigm change, as in the case of the discovery of oxygen. However it is not always the paradigm theory that is violated by new discoveries. Sometimes, as in the case of x-rays, it is the assumptions underlying the instruments employed in paradigmatic experimentation that can lead to paradigm-upheaval.

6. As Kuhn points out in his discussion of the discovery of X-rays, this time dimension can be a matter of only weeks. And it may actually be less than that. 


\section{Issues with Kuhn's Account of Discovery}

Let us first of all note that one need not subscribe to Kuhn's idea of paradigms or even any of Kuhn's more radical ideas (such as incommensurabilty), in order to adopt his distinction between that-what and what-that discoveries. Rather, one may well restrict oneself to talk about conceptual resources, accepted theories, background theories, etc. One may then speak of accepted background theories, rather than paradigms, against which some evidence is novel or unexpected. ${ }^{7}$ This is what we will do for the rest of this paper.

I believe Kuhn got many things right about the nature of discovery. First, I think it is reasonable that scientific discoveries of natural kinds must involve at least some degree of discovery-what. Consider the discovery of electrons. Electrons are generally considered to have been discovered by J.J. Thomson in I 897 . Obviously, electrons cannot be observed directly. But there were many physicists before Thomson who observed electrons as directly as one can, namely in the form of cathode rays. If the discovery-that were sufficient for the discovery simpliciter, the German physicist Johann Hittorf, who was the first to observe cathode rays in I869, would have to be deemed the discoverer of electrons. Second, if both the discovery-that and the discovery-what are required for the discovery simpliciter, then it makes sense to think that there are two kinds of discovery, resulting from the temporal order of those two aspects. Third, if the discoverythat of $\mathrm{X}$ precedes the discovery-what of X, i.e., if the discovery-that of $\mathrm{X}$ is unexpected, then the discovery simpliciter of $\mathrm{X}$ must take time, for it takes time to develop the conceptual resources accommodating X. Accordingly, Kuhn is right to say for those kinds of discoveries that they are "not isolated events, but extended episodes" (I996: 52). In contrast, if the discovery-what of X precedes the discovery-that of X, the discovery simpliciter can be instaneous, judged from the incidence of the discovery-that. Fourth, it is plausible to think that since in that-what discoveries the discovery-that cannot coincide with the discovery simpliciter, we cannot identify the discoverer-that with the discoverer simpliciter. Here too I agree with Kuhn. ${ }^{8}$ But that does not imply that we cannot identify the discoverer(s) of the that and the what aspects of a scientific discovery. I think we can. Fifth, it is plausible to think that that-what discoveries have the potential to excite or disturb the scientific community to a much greater extent than whatthat discoveries, where the discovery of $\mathrm{X}$ is anticipated. ${ }^{9}$

7. See also (Musgrave 1974).

8. In the converse case, we may very well do so. See section 6.3.I.

9. This seems to contradict the wide-held belief amongst philosophers of science that novel success ought to impress us much more than accommodative success. The historical evidence about scientists' attitudes, however, does not really support this belief. See (Schindler 20I3 $\mathrm{C}$ ). I thank one of the anonymous referees for raising this issue. 
The main criticism I have of Kuhn's account concerns the discovery-what. Kuhn implicitly assumes that the discovery-what of X must involve the correct conceptualisation of $\mathrm{X}$. He denies Priestley and Lavoisier the title of discoverer because neither of them had a correct conception of oxygen. Yet it remains unclear how correct one's conception of X must be. ${ }^{\text {IO }}$ It surely cannot be demanded that the discovery-what be an entirely correct conception of X. Consider again the example of the discovery of the electron. Since around 1927 we have known that electrons have wave-like properties. J.J. Thomson did not know that. So if we made the demand that one's conception of X needs to be entirely correct for one to be the discoverer of X, Thomson would not count as the discoverer of the electron. So the correct account of discovery must allow for at least some false and incomplete beliefs about $\mathrm{X}$ on the part of a person in order for that person to be able to qualify as the discoverer of $\mathrm{X}$. Let us refer to this problem of Kuhn's account as what-indeterminacy. I will offer a solution to this problem in section 5 .

Another qualm concerns the descriptive support for Kuhn's account. Peculiarly, Kuhn discusses no what-that discoveries at all. And I do not think that the example of a that-what discovery that he does discuss at some length supports his account.

Why is Priestley not the discoverer of oxygen? Because he believed he had discovered de-phlogisticated air, not oxygen. He thus did not have the right conception. Yet Kuhn gives another reason why Priestley cannot count as the discoverer. As we noted above, Kuhn has it that Priestley cannot be the discoverer of oxygen because he managed to isolate only an impure sample of oxygen. But if that were sufficient for a discovery of oxygen, then oxygen would have been

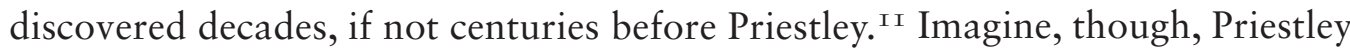
had managed to isolate a pure sample of oxygen. Would that have made him the discoverer of oxygen? Presumably not, since for that a correct conceptualisation is required.

In the following two sections, I will provide both an example for a what-that discovery that fills the gap of supporting Kuhn's claim about those kinds of discoveries and for a that-what discovery that is not subject to such technological contingencies as Kuhn's oxygen example.

Before engaging with the details of the two examples, let me make a more general remark. Although the ambition for a good account of scientific discovery (of natural kinds) should be for it to cover as many scientific discoveries (of natural kinds) as possible, it would be naïve to believe that any one account will cover all examples. It would be an advance though, I believe, to have an account that sheds light on many, or at least on some, examples. At this point, it is entirely open how

I0. This issue was first noted by Hudson (200I). See below for further details.

II. Both (Musgrave I976) and (Hudson 200I) repeat this argument uncritically. 
many examples the account proposed here can accommodate. Yet the two examples of the following sections, I believe, are very clear support for the account. ${ }^{\mathrm{I} 2}$

\section{Descriptive Support for Kuhn's Distinction}

\section{I. A That-What Discovery: the Discovery of the Zebra Pattern}

During the I960s, a major scientific revolution took place: a shift from a static image of the Earth's surface to a dynamic one. According to plate tectonics, the then new paradigm of geology, the Earth's surface consists of seven to eight major earth plates which move against each other, causing Earth quakes, volcanic activity, mountain-building, and tsunamis. Instrumental in instigating this revolution were patterns of magnetic anomalies first detected on the sea floor of the Eastern Pacific Ocean, just off the west coast of the USA, by the English geophysicist Ronald Mason and his assistant Arthur Raff, both working at the Scripps Institution of Oceanography at that time (Mason I958; Mason and Raff I96I; Raff I96I). These patterns consisted of positive and negative anomalies (with respect to the current direction of the geomagnetic field) ranging in broad bands from north to south along the ocean ridges. Mason and Raff described these patterns in terms of a "north-south magnetic lineation" (Raff I96I) and identified the positive anomalies as "the most striking feature of the [magnetic] map" (Mason I958: 328). Initially the origin of these patterns was ill-understood; the "significance of the lineation" was described as a "mystery" (Raff I96I: I48). Several geological models, assuming basalt rocks of varying size and shape underneath the sea floor, were taken into consideration (Mason I958; Mason and Raff I96I; Raff I96I). Yet these models were deemed unrealistic, for they implied topographic and seismic expression where none was found (Mason and Raff I96I). Rather surprisingly, all of these models sought to account for only the positive anomalies and completely ignored the negative anomalies (Schindler 2007). The conceptual and descriptive confusion surrounding these patterns started to be regimented only with the arrival of the so-called Vine-Matthews-Morley (VMM) hypothesis. The VMM hypothesis combined the sea floor spreading hypothesis (independently proposed in I96I-2 by H. Hess and R.S. Dietz), which had it that ocean floors were continually produced by molten rock escaping the Earth's mantle along the ocean ridges, with the idea that the north and south-poles of the Earth's magnetic field would

I2. This may in fact not be so different from physics where some (highly idealized) exemplars justify the theory and more complex situations (such as a falling leaf) are up for grabs (Cartwright I983). The relationship between philosophical theorizing and historical case studies is of course a complex one which cannot be discussed in any detail here. See my (Schindler 20I3b) and (Schindler 20I3a). 
switch throughout the Earth's history. As a result of these two processes, a pattern of magnetic anomalies would result on the sea floors (captured by the magnetites contained in the emerging molten rock) with positive and negative anomalies alternating. With the positive anomalies being shaded black and the negative anomalies being left white on maps, these patterns would later be referred to as 'zebra patterns' of magnetic anomalies, for obvious reasons. The fact that the sea floor spreading hypothesis only later became part of the correct explanation for the patterns of magnetic anomalies can be viewed as major reason for why the negative anomalies were simply ignored: they did not fit the conceptual presuppositions of the time, of which sea floor spreading was no part (Schindler 2007).

With respect to our discussion of discovery we ask: When and by whom was the zebra pattern of magnetic anomalies discovered? Clearly Mason and Raff first detected the pattern already in 1958 . They thus can be said have discovered that the zebra pattern of magnetic anomalies exists. But obviously we should resist saying that they discovered the zebra pattern of magnetic anomalies: they not only misdescribed it but also failed to take into consideration an essential part of the pattern (namely its negative anomalies). On the other hand, Vine, Matthews, and Morley, although clearly correctly describing and identifying the pattern in I963, did nothing by way of discovery-that, which had already occurred five years before. Kuhn's characterization of that-what discoveries thus appears to be vindicated: since the discovery-that preceded the discovery-what, the discovery necessarily had to take time. Given that the right conceptual resources were not in place when the discovery-that was made, it just had to take time before the discovery simpliciter could be made (together with the discovery-what). The discovery simpliciter, Kuhn is right, can therefore not be precisely identified as an event in time. And given that in this case the discovery-that and the discovery-what were made by different individuals, we cannot identify a discoverer (simpliciter) of the zebra pattern either, because that would require that there would have been an individual who discovered that and discovered what.

The discovery of the zebra pattern is a rather instructive example. Whereas Kuhn's example of the discovery of oxygen partially hinges on the (in this context) uninteresting contingency of technological capability, the discovery of zebra pattern does not. Here part of the data was systematically neglected, because it could not be accommodated on the basis of the conceptual resources at the time. And that - contrary to technological contingency-is informative, because that is, after all, how the two kinds of discovery are distinguished: in terms of the availability of those conceptual resources that render a certain object expected, or not.

\subsection{A What-That Discovery: the Discovery of the Higgs Boson}

The electroweak model proposed by Glashow (I96I), Weinberg (I967), and Salam (I968), which was going to form the basis of what we now call the Standard Model 
of fundamental particle physics, predicted the existence of the Higgs boson on the basis of the Higgs mechanism (developed already in 1964 by Peter Higgs and others in response to the troublesome Goldstone theorem in the context of symmetry breaking in strong interactions). ${ }^{\mathrm{I} 3}$ It was not before t'Hooft (I97I) showed that the model was renormalizable that this prediction was taken seriously. And, given the enormous technological know-how, effort, and financial backing required, it took exceedingly long for physicists to actually embark on probing the existence of the Higgs. Very recently, experiments carried out in the Large Hadron Collider at CERN first confirmed Higgs-like particles (July 20I2) and then its essential properties, such as zero spin and positive parity (March 20I3). The discovery of the Higgs boson undoubtably is to be viewed as a what-that discovery: it was a clearly anticipated discovery with all the required conceptual resources in place before any relevant observations were (or could be) made.

Kuhn's characterization of a what-that discovery seems to be correct in at least one important respect. The discovery of the Higgs boson was an "occasion only for congratulations, not for surprise" (I996: 56). This aspect is in fact one that been much remarked upon. For example the physicist Sean Carroll (CalTech), has recently stated that

It's a bittersweet victory when your theory turns out to be right, because it means, on the one hand, you're right, that's nice, but on the other hand, you haven't learned anything new that's surprising [emphasis added]. (in Heilprin 20I3)

In contrast, discovering a Higgs-like particle inconsistent with the predictions of the standard model would have given rise to the opportunity for exploring other theoretical options. As Stephen Hawking put it in a recent BBC interview, the discovery of the Higgs is "a pity in a way, because the great advances in physics have come from experiments that gave results we didn't expect."(BBC) Another Caltech physicist, Prof. Maria Spiropulu, was quoted by the New York Times as saying: "I personally do not want it to be standard model anything. I don't want it to be simple or symmetric or as predicted. I want us all to have been dealt a complex hand that will send me (and all of us) in a (good) loop for a long time" (in Overbye 2OI2). Finally, Jonathan Ellis, a physicist at CERN is on record for saying before

I3. The Goldstone theorem states that symmetry breaking must be accompanied by the appearance of a massless particle. Higgs managed to evade the Goldstone theorem by designing a field-theoretic model in which a massless photon produced by the symmetry breaking gained mass by "eating up" one of the two scalar particles inserted into the Lagrangian. The remaining scalar particle is the Higgs. Steven Weinberg and Abdus Salam, independently of each other, incorporated the Higgs mechanism into the model of leptons which Glashow had developed earlier (apparently initially unbeknownst at least to Weinberg). See Pickering (I984) for details. 
the discovery that, had the discovered boson the right mass: "This would be the real five star disaster, because that would mean there wouldn't need to be any new physics" (in Cho 2007).

Interestingly, there also seems to be a time-dimension to the Higgs discovery, seemingly contradicting Kuhn's characterization of what-that discoveries: although some first confirmatory evidence was announced in July $20 \mathrm{I} 2$ it took almost another year for physicists to be more confident to say that the discovered particle was actually the Higgs boson. Before we can address this apparent difficulty for Kuhn's account, we must first address the problem of what-indeterminacy (cf. section 3).

\section{Discovery as Essence Identification}

Although Kuhn clearly demands that correct conceptualisation is necessary for discovery, he does not specify how correct that conceptualisation needs to be. I propose the following account of discovery in order to fix this problem:

A discovery of $\mathrm{X}$ requires observing $\mathrm{X}$ or its direct effects (the discoverythat) and the correct conceptualisation of those of X's essential properties that suffice (epistemically) to individuate $\mathrm{X}$ at time $t$ (the discovery-what), whereby I take essential properties of $X$ to be those properties of $X$ that are (metaphysically) individually necessary and jointly sufficient for X. ${ }^{\mathrm{I}} 4$

In contrast to the alternative accounts of discovery to be discussed below, this account (just like Kuhn's) is an account intended to capture the nature of discovery, rather than to disambiguate priority debates. It therefore does without any reference to people making the discovery. Regardless, the account has implications for the requirements we set for someone to be a discoverer. In particular, on my proposal one may have a number of false beliefs about $\mathrm{X}$ and still count as the discoverer of $\mathrm{X}$ so long as one manages to correctly identify the essential properties of $\mathrm{X}$ sufficient for the individuation of $\mathrm{X}$ at the time.

To see how this proposal solves the problem of what-indeterminacy, let us consider once more the example of the discovery of the electron. Presumably there is a (time-independent) list of properties that are (metaphysically speaking) necessary and sufficient for the individuation of electrons. The list will involve negative charge, wave-particle duality, the charge to mass ratio, and spin. We may or may

I4. Essences need not be intrinsic but may be relational or historical (Okasha 2002; LaPorte 2009). Anti-essentialists may plug in their preferred notion of natural kinds here. What really does matter for the purposes of this paper is the point that there be some essential properties that suffice for the individuation of an object $\mathrm{X}$ at time $t$ relative to our background knowledge. 
not know all of those properties. We simply do not know, and we will probably never know. Our ignorance, however, does not prevent us from judging a discovery claim relative to the list of essential properties we do know, or have good reason to believe to be essential.

My account is a time-dependent one. That is, at one particular point in time, not all properties will be necessary for the individuation of natural kinds. Which of their properties are necessary will depend on the state of the background knowledge. For example, at the time when J.J. Thomson discovered the electron in I897, no other elementary particles with negative charge were known. So his discovery of a negatively charged particle was sufficient for him to be rightly regarded the discoverer of the electron. No successful identification of any of the other essential properties of electrons was required at that time for a legitimate discovery claim. Compare this to someone now claiming to have discovered a new negatively charged particle who had detected no other property than negative charge. Then, obviously, this would be insufficient for her to rightly claim to have discovered a new particle, for we know that there are many other negatively charged particles (apart from the electron: muon, tau, $\mathrm{W}^{-}$, and the negatively charged quarks).

With this new framework in hand, let us now turn again to the discovery of the Higgs boson. In the last section, we saw that the Higgs discovery appears to have a time dimension, despite the Higgs discovery clearly being a what-that discovery. In July $20 \mathrm{I} 2$ there was some evidence for the Higgs, but it was only in March $20 \mathrm{I} 3$ that the Higgs particle prediction by the standard model was fully confirmed.

With my proposed solution to the what-indeterminacy, we can now address this issue. What physicists discovered in July $20 \mathrm{I} 2$ were only Higgs-like particles. Such particles were compatible with the predictions of theoretical alternatives to the standard model, namely super symmetry models. In other words, physicists in July $20 \mathrm{I} 2 \mathrm{had}$ not yet ascertained enough properties sufficient to individuate the Higgs boson as predicted by the standard model. Only after they had managed to also detect the properties of spin-zero and positive parity were they able to say, by March 2013, that they had discovered the Higgs. Once those properties were detected, the discovery was 'instantaneous'. We can therefore precisely date the discovery of the Higgs particle as predicted by the standard model (some time in) March 20I3, and not before. Kuhn's account is therefore very much compatible with this discovery.

But, one may ask, would it not be more appropriate to describe the Higgs discovery as a what-that-what discovery? After all, identifying the essential properties of the Higgs boson required a determination of what properties (of the particle detected by the standard model) were detected at CERN. ${ }^{15}$

What-that-what discoveries, or discoveries involving even longer strings of

I 5. I thank one of the section editors of this journal for raising this question.

Ergo $\cdot$ vol. 2, no. 6 2015 
discoveries-that and discoveries-what (perhaps in different permutations) for that matter, are not possible on my account. Take the what-that-what triplet. The first part of that triplet would mean that there is a theory $\mathrm{T}$ that predicts an object $\mathrm{O}$ (as an instance of a natural kind), $\mathrm{O}$ was discovered, and $\mathrm{O}$ was exactly as predicted by $\mathrm{T}$. The second part of the triplet would mean that that same object $\mathrm{O}$ was causing conceptual problems that had to be accommodated by a reconfiguration of conceptual resources. But either $\mathrm{O}$ is exactly as predicted by $\mathrm{T}$ or it is not. In the former case, there will not be any reconceptualisation, so there will not be the second part of that triplet. In the latter case, there will be reconceptualisation, but only because $\mathrm{O}$ was not as predicted by $\mathrm{T}$. Hence there will not be a first part of the triplet. Thus, the Higgs boson is either a what-that discovery or a that-what discovery.

As it stands, our discovery of the Higgs is a what-that discovery (for the reasons mentioned above). But sure enough, this could turn out to be the wrong view of things. We might discover more properties of that particle in the future that are not consistent with the standard model. Those properties might be entirely unexpected or they might be exactly as predicted by the competitor theories of the standard model, namely supersymmetry theories (SUSYs). In the former case, we would have a that-what discovery after all. In the latter case, we would again have a what-that discovery (although now of a different sort). ${ }^{16}$ I shall discuss further aspects of the Higgs discovery in section 7.3.I.

Let us lastly also reconsider the discovery of oxygen. Although Priestley's conception of the gas he had discovered ('dephlogisicated air') was clearly false, arguably not everything about his conception was false. Minimally, Priestley got right that a certain class of chemical reactions are "bound together" and "viewed as results of a common underlying process", which is reversible (Carrier I991: 32). Today, we understand these reactions as instances of redox reactions, where elements are oxidised and reduced. ${ }^{17}$ The question is of course: are these properties of redox reactions enough for us to say that Priestley discovered oxygen? On the face of it, it looks as though these relations are to be treated as bare empirical relations associated with oxygen. In that case Priestley discovered that, but not what. And

I6. In that latter case, the discovery would still be surprising, despite being a what-that discovery. It would be surprising, because the well-confirmed standard model would be wrong. It would not be surprising in the sense of being entirely unpredicted, as with the some of the that-what discoveries mentioned above.

I7. It should be noted here in passing that there have been voices suggesting that the success of the phlogiston theory can be explained in modern terms by straightforward "correspondence relations", so that, for example, "dephlogistication of X corresponds to . . . a donation of electrons of $\mathrm{X}$-atoms to their bonding partner in the formation of a polarized or ionic chemical bond" (Schurz 20II). See also Schurz (2009). The first suggestion to this effect was made by Carrier (I99I: 32). See also Carrier (2004). Yet clearly neither Priestley nor Lavoisier had any conception whatsoever of electrons, let alone of their behaviour. 
although Lavoisier's conception was not entirely correct, Lavoisier not only correctly individuated some of oxygen's characteristic reactive behaviour (as Priestley had done), but he also (in contrast to Priestley) individuated oxygen's essential property of its being a chemical substance with a particular mass. ${ }^{18} \mathrm{He}$ thus may be rightly said to have discovered what oxygen is.

\section{Discovering Other Entities}

My discussion so far has been restricted to the discovery of natural kinds. One may wonder, though, whether the account is also applicable to the discovery of other entities than natural kinds. Consider the discovery of America. Christopher Columbus discovered that there was another continent hitherto unknown. To the end of his life, however, he was unaware of what he had discovered. He was firm in his belief that he had discovered a new sea route to the Far East and mistook Cuba for Japan. Interestingly, the cartographer Martin Waldseemüller, who first put the new continent on the map in I 507, decided to name it not after Columbus but rather after Amerigo Vespucci, who was the first to have realized what had been discovered, namely a new continent! Waldseemüller wrote:

I do not see what right any one would have to object to calling this part, after Americus [Vespucci] who discovered it [emphasis added] and who is a man of intelligence, Amerige, that is, the Land of Americus, or America: since both Europa and Asia got their names from women.

Within the Kuhnian framework we can explain why Waldseemüller would attribute the discovery to Vespucci: he discovered what America was (namely a new continent). And on the Kuhnian account, there is no discovery simpliciter without a discovery-what. But of course it was Columbus, not Vespucci, who discovered that there was a new continent. The discovery of America could thus be viewed as a that-what discovery whereby Columbus discovered that there was a new continent and Vespucci what that new continent was. The discovery simpliciter, in accord with Kuhn's account, is an extended period and cannot be attributed to either one individual alone. So the Kuhnian framework may very well apply also

I8. As mentioned above (Section 2) Lavoisier thought of oxygen as a 'principle' that would combine with (non-existent) caloric to form oxygen gas. Chang (2OI2), in the most recent contribution to the vast amount of literature on the overthrow of the phlogiston theory by the oxygen theory, defines principles as "fundamental substances that impart certain characteristic properties to other substances". Principles, in contrast to "other substances", were considered "active" in the sense of "effecting transformation of substances" (38). Chang also suggests that Lavoisier used the term "principle' almost synonymously with "element"' (6I). 
to entities other than natural kinds after all. How far it may carry, however, is of course an open question and cannot be decided here.

\section{Alternative Accounts of Scientific Discovery}

Since Kuhn proposed his account of scientific discovery there have been several alternative proposals. I will review these accounts in the following and briefly discuss their shortcomings. Some of these alternatives reject Kuhn's idea that either a discovery-that or a discovery-what alone is insufficient for a scientific discovery simpliciter. Accordingly, they also reject Kuhn's idea that it is always impossible to pin down the precise date of a discovery simpliciter. Perhaps even more importantly, they have not recognized that one should distinguish between two kinds of discovery (that-whats and what-thats), and seek to fit all scientific discoveries into a single procrustean bed.

\section{I. Achinstein's Observing X or its Direct Effects}

According to Achinstein (200I), a discovery has three components: an ontological, an epistemic, and a "priority" component. First, discovering X requires that $\mathrm{X}$ exists; one cannot discover pixies, unicorns, or hobbits. Second, in order to be identified as the legitimate discoverer of X, one must know that X exists. And for someone to know that (the object) X exists in the context of scientific discovery, for Achinstein, essentially amounts to "observations of the object itself or its direct effects" (200I: 269). Achinstein also speaks of an "epistemic situation necessary for discovering $X$ ". A person is in such a situation if "observations of X or its direct effects caused, or are among the things that caused, that person to believe that $\mathrm{X}$ exists, and among that person's reasons for believing that X exists is that X or its direct effects have been observed", or more briefly, "a person knows that X exists from [emphasis added] observations of X or its direct effects" (Achinstein 200I: 269). Achinstein dismisses the view that for a person $P$ to know $X, P$ must have only or mostly true (or substantially true) beliefs about X. Discoverers, although having some true beliefs about $\mathrm{X}$, regularly have also (many) false beliefs about $\mathrm{X}$ (Achinstein 200I: 274, 276-7). Third, for someone to be the discoverer of X, one must be the first (in some group of individuals) to know X. Achinstein combines these three components in the following definition:

$\mathrm{P}$ discovered $\mathrm{X}$ if and only if $\mathrm{P}$ was the first person (in some group) to be in an epistemic situation necessary for discovering X (200I: 269). 
What is striking about this definition is this: it says nothing about what the conditions are for someone to be in an epistemic situation that is sufficient for discovering $\mathrm{X}$. That is, there may be more involved in discovering $\mathrm{X}$ than merely (first) observing X or observing the direct effects of X. But Achinstein says nothing about those further conditions. All the same for Achinstein: for $\mathrm{P}$ to be the discoverer of $\mathrm{X}$ it is necessary and sufficient that $\mathrm{P}$ be in an epistemic situation merely necessary (but not necessarily sufficient) for discovering $\mathrm{X}$, i.e., that $\mathrm{P}$ observe $\mathrm{X}$ or its direct effects.

Achinstein for example identifies J.J. Thomson as the discoverer of the electron (in I 897). Although Thomson had many false beliefs about what electronswhich he referred to as 'corpuscles' - are (he thought they had no inertial mass, had stationary positions in atoms, were the only constituents of atoms, had no wave-like properties, were not carriers of the smallest electric charge) (Achinstein 200I: 274), he did have some important true justified beliefs about electrons. In particular, Thomson knew that cathode rays contain charged particles, that the ratio of mass to charge of the particles is approximately $\mathrm{IO}^{-7}$, and that those particles were much smaller than ordinary molecules (Achinstein 200I: 278). And because he knew these facts about electrons, and because knowledge of these facts about electrons implies that electrons exist, Achinstein reasons, we can legitimately say that Thomson is the discoverer of the electron (despite using the term 'corpuscle' rather than 'electron'):

If in I 897 Thomson knew that cathode rays contain charged particles, whose ratio of mass to charge is $1 \mathrm{O}^{-7}$ and that are much smaller than ordinary molecules, then I think it is reasonable to say that in I 897 he knew that electrons exist [. . . He knew of the existence of things that happen to be electrons. Electrons are the charged particles in question. Knowing these particular facts about them entails knowing that [electrons] exist. (2001: 279)

Whether or not Thomson really was the first to have been in an epistemic situation necessary for discovering the electron, Achinstein leaves open (200I: 279-80).

\section{I.I. Discussion}

In spite of what Achinstein claims, his account is arguably too weak to determine Thomson as (one of the) first discoverer(s) of the electron. Reconsider the 'facts' about electrons that Achinstein mentions: cathode rays contain charged particles, the ratio of mass to charge of the particles is approximately $\mathrm{IO}^{-7}$, and those particles are much smaller than ordinary molecules (200I: 278). These 'facts' about electrons are neither observable 'facts' nor are they facts having to do with the 
direct effects of electrons. They require an inference-to-the-best-explanation from the direct observable effects to there being electrons. And such an inference, in turn, arguably requires not only a discovery-that but also a discovery-what. But the latter plays no part in Achinstein's account. Again, for Achinstein, observing X or its direct effects is necessary and sufficient for us to say that $\mathrm{P}$ is the discoverer of X.

Moreover it is not clear at all on Achinstein's account how many observations of direct effects of $\mathrm{X}$ one must make in order to qualify for being worthy of being called a discoverer. As Achinstein mentions himself in passing, there were others, such as Hertz, Plücker, Perrin, Schuster, Lenard and Wiechert, who, long before Thomson, made some observations of the direct effects of electrons (200I: 279). Why do they not qualify as discoverers of the electron? What was it about their epistemic situation that was not sufficient for discovering electrons? Achinstein, although being adamant that they at best contributed to allowing Thomson to be in an epistemic situation necessary for discovering electrons (200I: 276), provides no answers.

On my account, Thomson counts as the discoverer of electrons because he was the first to identify some of their essential properties, which were sufficient for individuating electrons as a natural kind at his time.

In which category does the discovery of the electron fall? Is it a that-what or what-that discovery? This is not so straightforward in fact. On the one hand, cathode rays clearly were discovered before they were conceptualised correctly. So in this sense, they are a clear example of a that-what discovery. And yet, there was a crucial property of cathode rays, namely their deflection in an electrical field, which only Thomson managed to detect. And interestingly, that detection was strongly theory-driven. ${ }^{19}$ As mentioned above though (at the end of section 3 ), it is not to be expected that all discoveries fall neatly into either one or the other category. What does matter is that there are some cases that can be illuminated by the account proposed here (such as the ones discussed in section 4.I and 4.2).

\subsection{Hudson's Materially Demonstrated Base Descriptions}

The expressed goal of Hudson is it to propose an account of discovery that outlines a "principled way of deciding when discoveries are made and by whom" (200I: 77). According to this account,

I9. Falconer concludes her extensive study of Thomson's work on cathode rays by saying that "Thomson arrived at his conclusion that cathode rays were negatively charged, subatomic particles, a month or two before [emphasis added] he deconstructed Hertz's electric deflection experiment" (I985: 287). That is, Thomson correctly conceptualized an essential property of electrons before he was able to detect that property. In that sense, Thomson's discovery was a what-that discovery. 
A discovery of object $\mathrm{X}$ occurs when an individual (I) provides what we have called a base description for $\mathrm{X}$ and (2) succeeds in demonstrating materially [emphasis added] that this base description is satisfied. (Hudson 200I: 79)

Furthermore, as part of a "common sense understanding regarding scientific discovery"

(3) the discoverer finds something which is novel relative to a particular social community (the novelty condition) and (4) the object described and materially demonstrated is, in fact, $X$ (the truth condition). (Hudson 200I: 79)

Let us focus on the first two conditions. A base description, in Hudson's use of the term, "is a description of the object that suffices to identify it: something that satisfies this description is the object being considered" (200I: 77). Hudson stresses that base descriptions provide only identification conditions, not identity conditions. He understands this to imply that a base description of object X may not be satisfied and $X$ be present, and vice versa, a base description of $X$ may be satisfied and $\mathrm{X}$ not be present. Yet, if the satisfaction of a base description of $\mathrm{X}$ is taken to suffice to identify $\mathrm{X}$, as suggested by the above remarks by Hudson, then the latter cannot be true. In another formulation, Hudson says, more cautiously, that a base description is "one that, in the normal course of affairs, is useful [emphasis added] as an indicator [emphasis added] of a particular object-a description such that, if satisfied, we would anticipate [emphasis added] the presence of the object" (2001: 87-8). Hudson illustrates the idea of a base description with the following example:

Near Sudbury, Ontario, there is an enormous Canadian Nickel that signals to visitors that they have arrived at Sudbury. The description, 'the town where there is a big Canadian Nickel, many feet high' is a base description for Sudbury. It serves to pick out Sudbury. But if we moved the Nickel to Toronto, Toronto would not become Sudbury, and Sudbury would not stop being Sudbury. Still, under normal circumstances, and most of the time, it serves to identify Sudbury, and quite usefully. (200I: 78)

Furthermore, there may be other base descriptions, which serve the purpose of identifying Sudbury just as well

For some people, a particular restaurant might serve this purpose, or some other road marker ... if while driving in northern Ontario Sudbury is the 
only town for miles around, then 'the first town I see' can stand as a base description for Sudbury and used in a discovery claim (200I: 78)

Hudson agrees with Kuhn that a discovery involves not only a discovery-that, but also a discovery-what, i.e., a conceptualisation of the object in question. Yet, he points out that Kuhn has "left us with the quandary concerning how well one must conceptualise the discovered object" (Hudson 200I: 78). It is for this reason, Hudson believes, that one cannot precisely date a discovery nor precisely identify the discoverer on Kuhn's account (200I: 86; yet cf. section 2 of this paper). ${ }^{20}$ At the same time, Hudson believes that Kuhn is right that some conceptualisation of the discovered object is required for someone to lay a legitimate discovery claim. For him, a discovery is a "reflective event, one that impresses itself upon the discoverer as significant and informative", which it could not do if the discoverer had not in a "minimal way conceptualised the discovered object" (Hudson 200I: 78). Hudson attempts to accommodate these two considerations with the following proposal: "To have discovered an object one need only possess enough [emphasis added] conceptual resources to recognize its presence in a fairly reliable manner, and such resources are what base descriptions provide for us" (200I: 78). For someone to discover X, however, it is of course not enough for that person to possess a base description of X. For example, it is not enough for someone to possess the base description, 'the town where there is a big Canadian Nickel, many feet high', in order for them to be the discoverers of Sudbury. Rather, one needs to "seek a situation where this base description is satisfied" (Hudson 200I: 79). This is the condition of material demonstration of a base description. In our example, one might materially demonstrate the base description of Sudbury by "pointing to the Nickel while passing it in a car" (Hudson 200I: 79). In a sense Hudson's material demonstration corresponds to Kuhn's discovery-that.

Base descriptions need not be accurate. They can be false "in certain respects" as long as they (under normal circumstances) suffice to identify an object (Hudson 200I: 88). Appealing to an example by Donnellan (I966), Hudson points out that one can successfully identify Tom at a dinner party with the description 'the man with the martini', despite Tom drinking only ginger ale in a martini glass (because he is driving). Yet base descriptions need to be somehow 'appropriate' or 'correct', particularly when it comes to determining someone as the discoverer of something. Whether or not a base description is appropriate appears to be straightforward matter: all those base descriptions of $\mathrm{X}$ are appropriate that, under normal circumstances, allow one to identify X successfully (Hudson 200 I: 88-9).

Hudson's account is intended as a general account that is supposed to apply

20. As mentioned in section $\triangle$, I agree with the first of Hudson's judgements. With the second, I do not. On Kuhn's account, the inability to identify the discoverer (simpliciter) of an object in thatwhat discoveries is just a reflection of that kind of discovery's intrinsic time-dimension. 
to both 'homely' examples like discovering Sudbury or one's grandfather's watch (200I: 80-2) and scientific examples. The only scientific example Hudson discusses at length is the discovery of oxygen, first highlighted by Kuhn (see above). According to Hudson, Priestley possessed the following base description of oxygen: 'a species of air, highly respirable and combustible, which is a constituent of common, atmospheric air' (200I: 82). This, according to Hudson, is a base description "because there is no other gas other [sic] than [emphasis added] oxygen which satisfies this description" (200I: 82). ${ }^{21}$ Having a correct base description, of course, satisfies only one of Hudson's requirements for a discovery. Additionally, a discoverer must be the first (relative to a social community) to materially demonstrate the base description, and the object described and demonstrated must indeed be the object in question (i.e., requirement 3,2 , and 4 , respectively). The above base description Priestley demonstrated, amongst other things, by showing that the "evolved gas is not carbon dioxide" and by "repeated applications of Priestley's 'nitrous air' test", which used to be a standard procedure for testing the 'goodness' of common air, but which Priestley realized produced unexpected results in the case of oxygen (Hudson 200I: 83; cf. Kuhn I996: 59-60). With regard to the question of whether Priestley was the first to have discovered oxygen, however, Hudson is slightly undecided. Although he concludes that "Scheele [rather than Priestley] is the discoverer of oxygen according to the criteria I [i.e., Hudson] have set forth", he is happy to concede that more historical work may be required to settle the question (Hudson 200I: 84-5). More crucially, Hudson thinks that Priestley's false beliefs about the gas that he isolated (recall, Priestley believed that he had isolated dephlogisticated air) are irrelevant to the question of whether he discovered oxygen: "Priestley's false beliefs on this matter do not affect the adequacy of his material demonstration - he still finds something (i.e. oxygen) that is a highly combustible, highly respirable component of common air" (200I: 87). In other words, Priestley is the discoverer of oxygen (even though perhaps not the first), because he had a correct base description and he demonstrated this base description materially, in a sufficient way. Hudson concludes, contrary Kuhn, that "discoveries have definite discoverers and discovery times" (200I: 9I).

\subsection{Discussion}

Although Hudson concedes that a discovery-that is not sufficient for a discovery simpliciter and that a discovery-what is also needed, his account of base descriptions is rather deflationary. Base descriptions are interchangeable. As long as they 'suffice' to identify or 'indicate' an object as that what it is, they are good base

2I. This remark suggests that this particular base description is necessary and sufficient for identifying oxygen. Yet this does not sit very well with Hudson's account of such descriptions, which are supposed to be replaceable and context- and person-dependent (see above). 
descriptions. It is interesting to note that the base descriptions that Hudson mentions are all descriptions of 'superficial' properties such as air being respirable, combustible, and so on. And presumably, the properties relevant to base descriptions in Hudson's sense cannot be essential properties, for then they would not be interchangeable, person- and context-relative, such as the identification of Sudbury via the giant Canadian Nickel or a famous restaurant (see above). Demonstrating such base descriptions, however, cannot be enough for someone to lay a legitimate discovery claim in the scientific context. For example, base descriptions of the relevant surface properties of electrons will suffice to identify cathode rays (e.g., their behavior in electric fields). Yet surface properties will not ever suffice to identify electrons. For that we need an account that requires the identification of essential properties, such as charge and the particulate and wave properties of electrons.

\subsection{McArthur's Mathematico-Theoretical Discoveries}

McArthur (20II) has recently discussed an interesting discovery: the Casimir effect. The Casimir effect was predicted by the Dutch physicist Henrik Casimir in I948 as a consequence of quantum mechanics. Roughly, Casimir predicted that even at absolute zero, a vacuum contains non-zero electromagnetic forces, due to the continuous appearance and disappearance of virtual particles. He predicted that these forces would at least in principle be measurable between two perfect plane mirrors facing each other at an extremely small distance. Only in I996/7 was the Casimir effect convincingly confirmed by the American experimental physicist Steven Lamoreaux. ${ }^{22}$ According to McArthur, the undisputed discoverer of the measured effect, curiously, is not Lamoreaux (who first observed the effect), but rather Casimir, that is, the person who 'merely' predicted the effect (20II). As other examples of the allegedly same kind, McArthur mentions the top quark and the Higgs boson (20II: 370). McArthur concludes that to "materially demonstrate" (borrowing from Hudson) a theoretical prediction "is not sufficient to claim credit as discoverer" (20II: 370). Rather, when deciding on who was the discoverer of an object, "account must be taken of ... a mathematically derived [emphasis added] base description of the discovered object's observable effects" (20I I: 370). In fact, he goes as far as saying that "after this confirmation of the effect, we can unproblematically identify Casimir as the discoverer of the effect [emphasis added]" (McArthur 20II: 374).

McArthur's account of discovery is a structural account, because the base descriptions for the identification of an entity are of a structural nature. With regard to the Casimir effect, McArthur writes:

22. There was some prior qualitative confirmation by experiments much earlier (Sparnaay I958). 
What is essential in Casimir's [mathematically derived] base description [of the effect], then, is not necessarily the interpretation of [sic] the explanation of the object or discovered effect, but the relations that the effect obeys, regardless of the explanatory interpretation. (20I I: 37I)

More generally, McArthur merely demands that a discovery identify "basic relations that are exploited in later experiments, not their explanatory interpretations of why the particular relations obtain" (20II: 37I). Siding with the structural realists, McArthur maintains that it is the merely the structural relations identified in a discovery that must survive theory change. McArthur is rather vague on what these structural relations are supposed to amount to, but he describes them as "equations that represent the experimentally exploitable dispositional properties" (20II: 375).23 In McArthur's example of the Casimir effect, however, the to-be-retained-structures amount to no more than "relations obtaining between measurable quantities" (2OII: 373 ), such as the relation describing the forces between reflective plates in vacuums at absolute zero (20I I: 37I). Thus, it appears to be merely the identification of empirical relations (via mathematized theory) that McArthur regards as being essential to a legitimate discovery claim.

\subsection{Discussion}

In spite of inviting us to take scientists' judgments about the Casimir effect at face value, McArthur provides little evidence for the claim that Casimir is considered the discoverer and not Lamoreaux. Yet, he suggests that the Higgs discovery is a similar example. So let us dwell on it a little.

Should Peter Higgs be regarded as the discoverer of the Higgs boson, rather than the physicists experimentally confirming Higgs' prediction? After all, the particle bears his name and not the ones of who discovered that the Higgs boson exists. The analogous point also seems to motivate McArthur's case for attributing the discovery to Casimir rather than to Lamoreaux. More important than the label might be the fact that it is Peter Higgs, together with François Englert, ${ }^{24}$ and not the CERN physicists finding evidence for the Higgs boson, who received this year's

23. With "dispositional properties" McArthur is referring to a term used by Chakravartty $(2003)$. A dispositional property of a gas, for instance, is its disposition to expand when heated. Dispositions do not always manifest themselves, but are arguably always there.

24. Several individuals "co-discovered" the Higgs mechanism (as acknowledged by the 2010 Sakurai Prize for Theoretical Particle Physics): Brout and Englert (publishing several months before Higgs), Guralnik, Hagen and Kibble (publishing slightly after Higgs). Brout died in 20 I I and was thus not eligible for the 2013 Nobel Prize (it is well known that the Nobel Prize is not awarded posthumously). Interestingly, whilst Higgs (I 964) was the first to explicitly draw attention to the possibility of a massive boson (after the introduction of a further mechanism "presumably related to weak interactions"), he did not specify the properties of the Higgs boson, as we know it today (such as its 
Nobel Prize in physics. Can we infer from these facts that the discovery-what, at least in this case, must receive more weight than the discovery-that? I think we should be careful with our conclusions here.

First of all, for all that we know, the reason why the Higgs particle carries Peter Higgs' name might have no other reasons than mnemonic ones: it has allowed easy reference to what has long been recognized as an important prediction of the Standard Model. And once a label has stuck, it is hard to replace it with a new one. Even if there were good grounds for replacing the label, there still is the practical difficulty of the discovery-that involving hundreds of individuals. Who of those should be the name-giver?

Second, the details provided by the Nobel Prize committee for why they awarded Englert and Higgs the Nobel Prize surely matter: "for the theoretical discovery of a mechanism that contributes to our understanding of the origin of mass of subatomic particles, and which recently was confirmed through the discovery of the predicted fundamental particle, by the ATLAS and CMS experiments at CERN's Large Hadron Collider" ("The Nobel Prize" 20I3). That is, Higgs and Englert received the Nobel Prize because they predicted the Higgs boson (based on the mechanism they "discovered") that was then subsequently discovered by the experimentalists at CERN. The Nobel Prize committee clearly acknowledged that the CERN physicists discovered that the Higgs boson exists. The reason why none of the CERN physicists were awarded the Nobel Prize probably has got no other reasons than practical ones: although hundreds of physicists were involved in the discovery-that of the Higgs (at more or less equal measure), the Nobel Prize can be awarded to maximally three people. And possibly, the Nobel Prize committee could not determine any experimental physicists whose contribution was more significant than any of their peers'. ${ }^{25}$

It should be noted that the practical difficulty of identifying the discovererthat of the Higgs boson is not to be considered problematic for Kuhn's account. After all, Kuhn's claim about what-that discoveries posing no severe obstacles to the identification of the discoverers concerns the principled obstacles that can be found in that-what discoveries. In the latter, it is hard to identify the discoverer(s), because, regularly, the individuals discovering that certain objects exist are not the ones that conceptualise those objects (sufficiently) correctly (as for example in the discovery of the zebra pattern of magnetic anomalies; see

spin-zero property). This was done by Weinberg (I967), when incorporating the Higgs mechanism into his electroweak model of leptons.

25. The Nobel Prize committee has awarded the Nobel Prize in Physics to experimental physicists who were part of big collaborations. Carlo Rubbia and Simon van der Meer received the I984 Nobel Prize for their discovery of the W and Z particles in I983. I thank Helge Kragh for pointing this out to me. 
section 5). This has got nothing to do with the practical difficulty of awarding the title of discoverer to hundreds of individuals participating in the discoverythat. Rather, the discovery of the Higgs is a what-that discovery where those discovering-that already had the right conception of the discovered object at their disposal and did not first need to develop one on the basis of the results gained in experiment (as in that-what discoveries). Hence, it is clear that, in the discovery of the Higgs boson, those who discovered that the Higgs boson exists thereby discovered (simpliciter) the Higgs boson.

\section{Conclusion}

This paper sought to vindicate Kuhn's view of the nature of scientific discovery in important aspects, to specify some vague aspects of his account, and to defend his view against alternative accounts of discovery. Kuhn was right about a number of things. He was right about scientific discoveries (of natural kinds) requiring both a discovery-that and a discovery-what. Alternative accounts which regard either the discovery-that or the discovery-what to be sufficient for a discovery simpliciter, as we have seen in section 7 , set the bar too low: they render scientists discoverers who are not.

Kuhn was also right about there being two basic kinds of discovery, which come with markedly different characteristics. In particular, whereas what-that discoveries may well be instantaneous (with regard to the moment of discovery-that), that-what discoveries have an intrinsic time dimension: it will take time to correctly conceptualise an object that has not been anticipated prior to its discovery-that. This explains why it is impossible to determine a precise moment in time in which a that-what discovery is made. It also explains why it is to some extent arbitrary to identify discoverers (simpliciter) in such discoveries. Despite these discoveries therefore being 'troublesome', they are the kinds of discoveries that have the potential to significantly alter our view of the world-quite in contrast to what-that discoveries, which, although important in their own right as integral parts of normal science activity, are "occasion only for congratulations, not for surprise".

One important aspect is left vague in Kuhn's account: the discovery-what. That is, although Kuhn clearly believed that the correct conceptualisation is required for a discovery, he did not specify how correct the conceptualisation must be. Demanding that the conceptualisation be entirely correct leads to implausible consequences (the electron was not discovered until only after the advent of quantum mechanics). Weaker requirements must be set. This paper proposed that for a discovery to be made, some essential properties of the object in question must be discovered (both with regard to the discovery-what and the discovery-that). That 
allows for the object in question possessing properties not recognized (or even not at the time recognizable) by the discoverer to be discoverable later in time, without casting undue skepticism on the original discovery.

The account presented here is an account that is meant to apply primarily to natural kinds. It may well be, though, that the account is more generally applicable than that.

\section{Acknowledgements}

I want to thank Asbjørn Steglich-Petersen, Jens Christian Bjerring, Theodore Arabatzsis, Mads Goddiksen, Sara Green, Robin Hendry, Brian Hepburn, Helge Kragh, James McAllister, Rune Nyrup, Wendy Parker, Mattias Rasmussen, Johanna Seibt, Asger Steffensen, Peter Vickers, and Joeri Witteveen (amongst others) for valuable feedback in discussion and the anonymous referees and the editors of this journal for their detailed comments on an earlier version of this article. Thanks also to Robert Mason for meticulous proof-reading.

\section{References}

Achinstein, Peter (200I). The Book of Evidence. Oxford University Press. http://dx.doi. org/10.1093/0195143892.001.0001

BBC (20I2, July 4). Stephen Hawking on Higgs: 'Discovery has lost me \$roo'. Retrieved from http://www.bbc.co.uk/news/science-environment-18708626.

Carrier, Martin (199I). What Is Wrong with the Miracle Argument? Studies in History and Philosophy of Science, 22(I), 23-36. http://dx.doi.org/10.1016/0039-3681(91)90013-I

Carrier, Martin (2004). Experimental Success and the Revelation of Reality: The Miracle Argument for Scientific Realism. In Martin Carrier, Johannes Roggenhofer, Günter Küppers, and Philippe Blanchard (Eds.), Knowledge and the world: Challenges beyond the science wars. Springer (137-16I).

Cartwright, Nancy (1983). How the Laws of Physics Lie. Oxford University Press. http:// dx.doi.org/10.1093/0198247044.001.0001

Chakravartty, Anjan (2003). The Structuralist Conception of Objects. Philosophy of Science, 70(5), 867-878. http://dx.doi.org/10.1086/377373

Chang, Hasok (20 2 2). Is Water $\mathrm{H}_{2} \mathrm{O}$ ? Evidence, Realism and Pluralism. Vol. 293, Boston Studies in the Philosophy of Science. Springer.

Cho, Adrian (2007). Physicists' Nightmare Scenario: The Higgs and Nothing Else. Science, 315(58I9), I657-I658. http://dx.doi.org/10.1126/science.315.5819.1657

Donnellan, Keith S. (1966). Reference and Definite Descriptions. The Philosophical Review, 75 (3), $28 \mathrm{I}-304$. http://dx.doi.org/10.2307/2183143

Falconer, Isobel (I985). Theory and Experiment in J.J. Thomson's Work on Gaseous Discharge (Doctoral thesis, University of Bath). 
Glashow, Sheldon L. (I96I). Partial-Symmetries of Weak Interactions. Nuclear Physics 22(4), 579-588. http://dx.doi.org/10.1016/0029-5582(61)90469-2

Hanson, Norwood Russel (I958). Patterns of Discovery: An Inquiry into the Conceptual Foundations of Science. University Press.

Heilprin, John (2013). Higgs Boson Discovery Confirmed after Physicists Review Large Hadron Collider Data at Cern. Huffington Post, I $4 / \mathrm{o}_{3} / \mathrm{I}_{3}$.

Higgs, Peter W. (I 964). Broken Symmetries and the Masses of Gauge Bosons. Physical Review Letters, I3(I 6), 508-509. http://dx.doi.org/10.1103/PhysRevLett.13.508

Hudson, Robert G. (200I). Discoveries, When and by Whom? The British Journal for the Philosophy of Science, 52(I), 75-93. http://dx.doi.org/10.1093/bjps/52.1.75

Kuhn, Thomas S. (I959). Energy Conservation as an Example of Simultaneous Discovery. In Marshall Clagett (Ed.), Critical Problems in the History of Science (32 I-356). University of Wisconson Press.

Kuhn, Thomas S. (I962a). Historical Structure of Scientific Discovery. Science, I36(35 I 8), 760-764. http://dx.doi.org/10.1126/science.136.3518.760

Kuhn, Thomas S. (I962b). The Structure of Scientific Revolutions. University of Chicago Press.

Kuhn, Thomas S. (I977). The Essential Tension. University of Chicago Press.

Kuhn, Thomas S. (I996). The Structure of Scientific Revolutions (3rd ed.). University of Chicago Press. (Original work published I962) http://dx.doi.org/10.7208/chicago/9780226458106.001.0001

LaPorte, Joseph (2009). Natural Kinds and Conceptual Change. Cambridge University Press. http://dx.doi.org/10.1017/CBO9780511527319

Mason, Ronald G. (I958). A Magnetic Survey Off the West Coast of the United States between Latitudes 32 and 36 N, Longitudes I2 I and I28 W. Geophysical Journal, I(4), 320-329.

Mason, Ronald G., and Arthur D. Raff (I96I). Magnetic Survey Off the West Coast of North America, 32 N. Latitude to 42 N. Latitude. Geological Society of America Bulletin, 72(8), I 259-I 265. http://dx.doi.org/10.1130/0016-7606(1961)72\%5B1259:MS OTWC\%5D2.0.CO;2

McArthur, Daniel J. 20I I. Discovery, Theory Change and Structural Realism. Synthese, I79(3), 36I-376. http://dx.doi.org/10.1007/s11229-009-9672-z

Musgrave, Alan (I974). Logical Versus Historical Theories of Confirmation. British Journal for the Philosophy of Science, I-23. http://dx.doi.org/10.1093/bjps/25.1.1

Musgrave, Alan (1976). Why Did Oxygen Supplant Phlogiston? Research Programmes in the Chemical Revolution. In Colin Howson (Ed.), Method and Appraisal in the Physical Sciences (I 8 I-209). Cambridge University Press. http://dx.doi.org/10.1017/ CBO9780511760013.005

Okasha, Samir (2002). Darwinian Metaphysics: Species and the Question of Essentialism. Synthese, I3I(2), I9I-2I3. http://dx.doi.org/10.1023/A:1015731831011

Overbye, Dennis (20I2). Physicists Find Elusive Particle Seen as Key to Universe. New York Times, 04/07/20I 2.

Pickering, Andy (1984). Constructing Quarks: A Sociological History of Particle Physics University of Chicago Press.

Raff, Arthur D. (I96I). The Magnetism of the Ocean Floor. Scientific American, 205, I46I 57. http://dx.doi.org/10.1038/scientificamerican1061-146

Salam, Abdus (1968, July I9-25). Elementary Particle Physics. In N. Svartholm, ed., Pro- 
ceedings of the Eighth Nobel Symposium, conducted at the meeting of The Nobel Foundation, Stockholm.

Scerri, Eric R., and John Worrall. 200I. Prediction and the Periodic Table. Studies in History and Philosophy of Science Part A 32 (3):407-452. http://dx.doi.org/10.1016/ S0039-3681(01)00023-1

Schindler, Samuel (2007). Rehabilitating Theory: Refusal of the 'Bottom-Up'construction of Scientific Phenomena. Studies in History and Philosophy of Science Part A, 38(I), I60-I 84. http://dx.doi.org/10.1016/j.shpsa.2006.12.009

Schindler, Samuel (2013a). History and Philosophy of Science: Coherent Programme at Last? Metascience, 22(2), 457-46o. http://dx.doi.org/10.1007/s11016-012-9728-4

Schindler, Samuel (2013b). The Kuhnian Mode of HPS. Synthese, I90(I8), 4I37-4I 54. Preprint available from http://dx.doi.org/10.1007/s11229-013-0252-x.

Schindler, Samuel (20I3c). Novelty, Coherence, and Mendeleev's Periodic Table. Studies in History and Philosophy of Science Part A, 45, 62-69. http://dx.doi.org/10.1016/j. shpsa.2013.10.007

Schurz, Gerhard (2009). When Empirical Success Implies Theoretical Reference: A Structural Correspondence Theorem. The British Journal for the Philosophy of Science, 6o(I), IоI. http://dx.doi.org/10.1093/bjps/axn049

Schurz, Gerhard (20I I). Structural Correspondence, Indirect Reference, and Partial Truth: Phlogiston Theory and Newtonian Mechanics. Synthese, I 80, I03-I 20. http://dx.doi. org/10.1007/s11229-009-9608-7

Sparnaay, M. J. (I958). Measurements of Attractive Forces between Flat Plates. Physica, 24, 75 I-764. http://dx.doi.org/10.1016/S0031-8914(58)80090-7

't Hooft, Gerald (I97I). Renormalizable Lagrangians for Massive Yang-Mills Fields. Nuclear Physics B, 35(I), I 67-I 88. http://dx.doi.org/10.1016/0550-3213(71)90139-8

The Nobel Prize in Physics 20I3 (2013, October 8). Retrieved from http://www.nobelprize. org/nobel_prizes/physics/laureates/2013/

Weinberg, Steven (1967). A Model of Leptons. Physical Review Letters, I9(2I), I 264. http://dx.doi.org/10.1103/PhysRevLett.19.1264 